\title{
Salicylic acid and salicylic acid glucoside in xylem sap of Brassica napus infected with Verticillium longisporum
}

\author{
Astrid Ratzinger $\cdot$ Nadine Riediger · \\ Andreas von Tiedemann · Petr Karlovsky
}

Received: 2 January 2009/Accepted: 5 April 2009/Published online: 16 May 2009

(C) The Botanical Society of Japan and Springer 2009

\begin{abstract}
Salicylic acid (SA) and its glucoside (SAG) were detected in xylem sap of Brassica napus by HPLCMS. Concentrations of SA and SAG in xylem sap from the root and hypocotyl of the plant, and in extracts of shoots above the hypocotyl, increased after infection with the vascular pathogen Verticillium longisporum. Both concentrations were correlated with disease severity assessed as the reduction in shoot length. Furthermore, SAG levels in shoot extracts were correlated with the amount of $V$. longisporum DNA in the hypocotyls. Although the concentration of SAG (but not SA) in xylem sap of infected plants gradually declined from 14 to 35 days post infection, SAG levels remained significantly higher than in uninfected plants during the whole experiment. Jasmonic acid (JA) and abscisic acid (ABA) levels in xylem sap were not affected by infection with $V$. longisporum. SA and SAG extend the list of phytohormones potentially transported from root to shoot with the transpiration stream. The physiological relevance of this transport and its contribution to the distribution of SA in plants remain to be elucidated.
\end{abstract}

Keywords Brassica napus - Phytohormones ·

Salicylic acid - Salicylic acid glucoside .

Verticillium longisporum $\cdot$ Xylem sap

\section{A. Ratzinger · P. Karlovsky ( $\square)$}

Molecular Phytopathology and Mycotoxin Research Unit, Department of Crop Sciences, Goettingen University,

37077 Göttingen, Germany

e-mail: pkarlov@gwdg.de

N. Riediger - A. von Tiedemann

Plant Pathology and Plant Protection Unit,

Department of Crop Sciences, Goettingen University,

Göttingen, Germany
Abbreviations
ABA cis-Abscisic acid
Dpi Days post inoculation
JA Jasmonic acid
$\mathrm{m} / \mathrm{z}$ Mass-to-charge ratio
SA Salicylic acid
SAG Salicylic acid glucoside

\section{Introduction}

Salicylic acid (SA) is a phytohormone involved in the control of plant defence against pathogens. The role of SA in the activation of systemic acquired resistance (SAR) has been proven on biochemical and genetic levels. Treatment with SA or its analogues enhances the resistance of plants against pathogens, while mutants with disrupted SA synthesis and plants engineered to hydroxylate SA enzymatically exhibit increased susceptibility to diseases (reviewed by Loake and Grant 2007). Conversely, plants expressing bacterial genes for SA synthesis acquired enhanced resistance to pathogen infection (Verberne et al. 2000). While the role of SA in SAR is undisputed, SA is not the primary factor that is translocated from the place of infection to distant organs where it triggers SAR (Rasmussen et al. 1991; Vernooij et al. 1994). The identity of the primary systemic signal has not yet been conclusively established.

The major nonvolatile conjugate of SA is glucoside (SAG). SAG is regarded as a storage form or detoxification product of SA. Accumulation of SAG in the vacuole (Dean et al. 2005), and the absence of SAG from the phloem sap of plants with induced SAR (Enyedi et al. 1992), supported this view. Further corroboration came from the observation of a slow, long-lasting defence-like response in tobacco 
suspension culture treated by SAG, which was explained by a gradual release of SA by SAG hydrolysis (Kawano et al. 2004). This view was challenged recently by the work of Umemura et al. (2008), who used RNAi-mediated silencing of the glucosyl transferase responsible for SAG formation and showed that the activity contributed significantly to SAR development. The authors suggested that glucosylation of SA may actually be a key mediator of SAR. Overexpression of SA glucosyl transferase in Arabidopsis led to contradictory results, since the levels of free SA and SAG (as well as the glucose ester of SA) decreased rather than increased (Song et al. 2008).

Salicylic acid derivatives can be transported over long distances principally via phloem or xylem. Phloem moves plant metabolites in the source-to-sink direction while xylem transport takes place along the transpiration stream. Solutes can thus be transported in opposite directions in xylem and phloem, depending on the ratio of photosynthetic and respiratory activities of the organs involved. In phloem, SA levels are elevated upon pathogen attack (Métraux et al. 1990; Yalpani et al. 1991). In cucumber, free SA is translocated by phloem from cotyledon to leaves (Mölders et al. 1996), but phloem transport of SA from an inoculated leaf is low (Rasmussen et al. 1991). Conjugated forms of SA were not found in phloem sap of infected tobacco plants (Enyedi et al. 1992). In contrast to phloem, until recently, only indirect evidence indicated that xylem may be involved in the transport of SA. The distribution of $\left[{ }^{14} \mathrm{C}\right]-\mathrm{SA}$ from an infiltrated leaf within the Arabidopsis rosette could not be explained by phloem translocation alone (Kiefer and Slusarenko 2003). Rocher et al. (2006) applied $\left[{ }^{14} \mathrm{C}\right]$-labelled SA exogenously to the cotyledons of castor bean and observed movement of radioactivity via phloem towards the basal part of the seedling, where part of it was transported into the xylem and carried back to the shoot. The authors suggested that long-distance transport of SA via xylem may contribute to SA accumulation in distal parts of the plant in response to infection of its lower parts, in addition to endogenous synthesis and allocation by phloem. Their attempts to determine endogenous SA in xylem sap of castor bean seedlings failed.

Verticillium longisporum is a soil-borne fungal pathogen with host specificity for the genus Brassica. The fungus enters host plants through their roots and colonises xylem vessels, to which it remains restricted for most its infection phase (Eynck et al. 2007; Zhou et al. 2006). The main motivation for the taxonomical separation of $V$. longisporum from the closely related $V$. dahliae is the ability of $V$. longisporum to infect crucifers. $V$. dahliae is therefore commonly used in studies of $V$. longisporum pathogenicity as a control to test for the specificity of the observed effects.
In this work we demonstrate that SA and SAG are present in xylem sap of Brassica napus plants, and show that the concentrations of both compounds increase following infection with Verticillium longisporum.

\section{Materials and methods}

\section{Plant material}

Rapid-cycling rape (Brassica napus var. napus, genome ACaacc) was originally provided by P.H. Williams (Department of Plant Biology, University of WisconsinMadison, WI; Crucifer Genetics Cooperative, Stock number 5 ). Seeds were surface sterilised by immersing them in $70 \%$ ethanol for $30 \mathrm{~s}$ and then rinsing with sterilised tap water for $30 \mathrm{~s}$ three times.

\section{Fungal isolates}

Verticillium longisporum VL 43 from B. napus and $V$. dahliae VD 73 from Linum usitatissimum were used in this study. Both strains were isolated from plants grown in northern Germany as described in Zeise and von Tiedemann (2001).

Fungal stocks with $1-3 \times 10^{6}$ conidia $\mathrm{ml}^{-1}$ were stored at $-80^{\circ} \mathrm{C}$ in $25 \%$ glycerol.

Preparation of inoculum for plant infection

Shake cultures were started by adding $500 \mu \mathrm{l}$ spore sus-

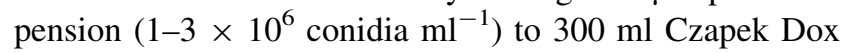
broth. The inoculated broth was incubated at $23^{\circ} \mathrm{C}$ on a rotary shaker at $100 \mathrm{rpm}$ in the dark. After 10-14 days, the culture was filtered through sterile gauze. Spore concentration was determined using a haemocytometer and diluted to $1 \times 10^{6}$ spores $\mathrm{ml}^{-1}$.

Plant inoculation and cultivation

Rapid-cycling rapeseeds were germinated in autoclaved sand in climate-controlled chambers (30 kLux, 60\% humidity, $22 / 20^{\circ} \mathrm{C}$ day/night, and 15 -h day length; Philips TL5 HO lamps). Seven-day-old seedlings were inoculated by root-dipping in a spore suspension of either $V$. longisporum (isolate $43,1 \times 10^{6}$ spores $\mathrm{ml}^{-1}$ ) or $V$. dahliae (isolate $73,1 \times 10^{6}$ spores $\mathrm{ml}^{-1}$ ) for $30-45 \mathrm{~min}$. As a control, seedlings were dipped in sterile water. After inoculation, single seedlings were potted in a sterile sand-soil mixture (1:1) and grown under the conditions described above. 
Xylem sap preparation

Rapeseed plants were harvested 28 days post inoculation (dpi). For time-course experiments, xylem sap was collected at 14, 21, 28, and 35 dpi. Shoots were cut above the hypocotyl, and xylem sap was collected by pressurizing roots to $4 \times 10^{5} \mathrm{~Pa}$ for $15 \mathrm{~min}$ using a Scholander pressure chamber (Scholander et al. 1965). Xylem sap was filtered through a $0.2-\mu \mathrm{m}$-Teflon filter (WICOM, Heppenheim, Germany) and subjected to HPLC-MS analysis immediately or stored at $-20^{\circ} \mathrm{C}$. Xylem sap was checked for contamination with cytoplasm by determining malate dehydrogenase activity (absence of activity indicated absence of cytoplasmic contamination).

Determination of sucrose concentration in xylem sap

The concentration of total sucrose and glucose in xylem sap was determined using the assay kit Enzytec fluid sucrose (r-biopharm, Darmstadt, Germany), which is based on enzymatic hydrolysis of sucrose, phosphorylation of glucose by the activity of hexokinase and oxidation of glucose-6-phosphate to gluconate-6-phosphate by glucose6-phosphate dehydrogenase. The increase in NADH concentration was monitored by UV absorption at $340 \mathrm{~nm}$ (Specord 40, Analytik Jena, Germany). The concentration of free glucose was determined using an Enzytec fluid D-Glucose assay kit (r-biopharm, Darmstadt, Germany), which is based on the same principle except for the omission of the sucrose hydrolysis step. Standard deviation of technical replicas was lower than $4 \%$ for both assays. The concentration of sucrose was determined by subtracting the glucose concentration from the sum of sucrose and glucose.

\section{Preparation of shoot extracts}

The shoots of plants from which xylem sap was obtained were shock frozen with liquid nitrogen and stored at $-80^{\circ} \mathrm{C}$. Frozen shoot material was lyophilized $\left(\right.$ at $\left.-20^{\circ} \mathrm{C}\right)$ and ground in a ball mill. Extraction was performed as follows: to $50 \mathrm{mg}$ dry shoot material (two replications for each plant), $1.5 \mathrm{ml}$ methanol (gradient quality; Fisher Scientific, Schwerte, Germany) and $1.0 \mathrm{ml}$ chloroform (p.a.; Roth, Karlsruhe, Germany) were added. The mixture was shaken vigorously and incubated for $5 \mathrm{~min}$ at $37^{\circ} \mathrm{C}$. Subsequently, $2 \mathrm{ml}$ either twice-distilled water or $2 \%$ formic acid (puriss. p.a.; Fluka, Seelze, Germany) in water were added. The suspension was shaken for $2 \mathrm{~h}$ at room temperature. The samples were centrifuged at $3,583 \mathrm{~g}$ for $15 \mathrm{~min}$, the phases were separated, and the polar phase dried under vacuum at $30^{\circ} \mathrm{C}$. The residue was dissolved in $1 \mathrm{ml}$ methanol/bi-distilled water (1:9), and the solution was filtered through a $0.2-\mu \mathrm{m}$-Teflon filter. Samples were directly subjected to HPLC-mass spectrometry (HPLCMS) or stored at $-20^{\circ} \mathrm{C}$.

High performance liquid chromatography and mass spectrometry

Methanol (gradient quality; Fisher Scientific, Schwerte, Germany), acetonitrile (gradient quality; VWR, Darmstadt, Germany), and acetic acid (p.a.; Fluka) were used for the mobile phases.

For full-scan analysis, an HPLC system equipped with autosampler (ProStar 410, Varian, Darmstadt, Germany), binary pump system (ProStar 210, Varian), degasser, and a column oven coupled to an ion trap mass spectrometer (500-MS, Varian) via electrospray ionisation source was used. Samples were injected onto a reversed-phase column Polaris C18-Ether $(100 \times 2 \mathrm{~mm}, 3 \mu \mathrm{m}$ particle size; Varian) kept at $40^{\circ} \mathrm{C}$. The mobile phase consisted of a binary gradient of $7 \mathrm{mM}$ acetic acid in $95 \%$ water with $5 \%$ acetonitrile (A), and $7 \mathrm{mM}$ acetic acid in methanol (B): 0.0-2.2 min 10\% B, 2.2-25.0 min from $10 \%$ B to $98 \%$ B, 25-30 min $98 \% \mathrm{~B}$, followed by washing $(98 \% \mathrm{~B})$ and re-equilibration $(10 \% \mathrm{~B})$ steps at a flow rate of $0.2 \mathrm{ml} / \mathrm{min}$. The electrospray ionisation source was operated either with negative or positive spray polarity with the mass spectrometer operating in a full scan mode at a mass range of a mass-to-charge ratio $(\mathrm{m} / \mathrm{z}) 100-1,000$ and a scan speed of $5,000 \mathrm{Da} / \mathrm{s}$. Raw data files were converted into NetCDF format and processed using XCMS Analyte Profiling Software (Smith et al. 2006).

Phytohormones, SA, and SAG were quantified using multiple reaction monitoring mode on the HPLC system specified above connected to a triple-quadrupole mass spectrometer (1,200 L, Varian) with an electrospray interface. Standards of SA, jasmonic acid (JA), and abscisic acid (ABA) were purchased from Sigma-Aldrich. Salicylic acid glucoside was kindly provided by H. Kneifel (Research Centre Jülich, Institute Phytosphere, Jülich, Germany). The analytical compounds were separated on a reversed-phase column (Polaris C18-A, $125 \times 2 \mathrm{~mm}, 5 \mu \mathrm{m}$ particle size, Varian) at $40^{\circ} \mathrm{C}$ and eluted isocratically with $7 \mathrm{mM}$ acetic acid in $47.5 \%$ water, $2.5 \%$ acetonitrile, and $50 \%$ methanol at a flow rate of $0.2 \mathrm{ml} / \mathrm{min}$. The eluate was subjected to negative electrospray ionization, and the ions were detected using the following mass transitions: SAG $\mathrm{m} / z 298.8 \rightarrow \mathrm{m} / \mathrm{z}$ 137 and $m / z 298.8 \rightarrow m / z$ 93; SA $m / z 136.8 \rightarrow m / z$ 93; ABA $m / z 262.8 \rightarrow m / z, 153$, JA $m / z 208.9 \rightarrow m / z 59$.

\section{Quantification of $V$. longisporum DNA}

Fungal biomass was determined in lyophilized hypocotyls from single plants harvested at 28 dpi. DNA was isolated using the cetyltrimethylammonium bromide (CTAB) 
method as modified by Brandfass and Karlovsky (2006), except for the final precipitation with polyethylene glycol (polyethylene glycol 6000, Serva, Heidelberg, Germany), which was performed as follows. After chloroform extraction, $700 \mu \mathrm{l}$ DNA solution in CTAB buffer was transferred to a $1.5-\mathrm{ml}$ tube containing $200 \mu \mathrm{l}$ polyethylene glycol (30\%) and $100 \mu \mathrm{l} 5 \mathrm{M} \mathrm{NaCl}$; the solution was mixed, incubated for $20 \mathrm{~min}$ at room temperature, and centrifuged for $15 \mathrm{~min}$ at $14,000 \mathrm{~g}$. The pellet was washed with $70 \%(\mathrm{v} / \mathrm{v})$ ethanol, dried, and dissolved in $30 \mu \mathrm{TE}$ buffer (10 mM Tris/HCl, $1 \mathrm{mM}$ EDTA, pH 8.0). DNA concentration was estimated by densitometry after electrophoresis in $0.8 \%(\mathrm{w} / \mathrm{v})$ agarose gels (Cambrex, Rockland, ME) in TAE buffer $(40 \mathrm{mM}$ Tris, $1 \mathrm{mM}$ EDTA, $\mathrm{pH} 8.5)$ at $7 \mathrm{~V} / \mathrm{cm}$ for $60 \mathrm{~min}$. Densitometry was performed as described previously (Brandfass and Karlovsky 2006). Fungal DNA was quantified by real-time PCR as described by Eynck et al. (2007).

\section{Statistics}

Data are presented as mean \pm standard deviation. The standard deviations for the increases in SA or SAG relative to the control were calculated according to the error propagation formula. Differences in concentrations of phytohormones and SA derivatives among uninfected, $V$. longisporum-infected, and $V$. dahliae-infected plants were determined by ANOVA, and the significance of correlation coefficients was determined with 1-tailed $t$ tests (SPSS 15.0; SPSS, Chicago, IL). All correlation coefficients presented in the figures are significant at the 0.05 level.

\section{Results}

Salicylic acid glucoside in xylem sap and shoots of B. napus

Xylem sap extracted from B. napus plants infected with $V$. longisporum and from healthy controls was analysed by HPLC-MS to identify metabolites affected by the infection. Plants at $28 \mathrm{dpi}$ were used because at this time the amount of xylem sap extractable from a single plant exceeded $100 \mu \mathrm{l}$. Xylem sap was filtered and subjected to HPLC-MS analysis directly.

The concentration of the sum of sucrose and glucose in xylem sap was determined in order to estimate the contamination of xylem sap with phloem sap. Xylem sap of infected plants contained $2.17 \pm 1.04 \mathrm{mM}$ sucrose plus glucose, and xylem sap of uninfected controls contained $3.10 \pm 0.31 \mathrm{mM}$ sucrose plus glucose, indicating that contamination of xylem with phloem sap was lower than $0.3 \%$ (see "Discussion" for calculation).
Comparison of the metabolic profiles of infected and healthy plants revealed a number of signals with intensities that were increased in infected plants. One of these signals had $m / z=299$ - the same as that of the molecular ion of SAG in negative ionisation mode.

Methanol/chloroform/water extracts were prepared from shoots of the plants from which the xylem sap originated. Metabolic profiles of the polar phase of these extracts were recorded in negative ionisation mode and examined for signals with $\mathrm{m} / \mathrm{z}$ of 299 . Three signals were found, the intensity of one of which increased after infection. At the retention time of the metabolite generating this signal (3.5 min), another signal at $\mathrm{m} / \mathrm{z}, 137$ was detected. Because 138 is the molecular weight of SA and the difference between 299 and 137 corresponds to a glucose residue, we hypothesised that the signal at $\mathrm{m} / \mathrm{z} 299$ originated from SAG, and that the ion with $\mathrm{m} / z$ of 137 originated from free SA generated during ionisation by in-source fragmentation. The hypothesis was proven correct by comparing the xylem metabolite with $\mathrm{m} / z, 299$ with chemically synthesised SAG. Comparison of retention times and analysis of product ion spectra on a triple quadrupole confirmed that the signal with $m / z 299$, which was enhanced by infection with $V$. longisporum, originated from SAG (Fig. 1). The extracted ion chromatogram for negative ion $\mathrm{m} / \mathrm{z} 299$ for xylem sap obtained from plants infected with the taxonomically related species $V$. dahliae, which is not a pathogen for B. napus, was indistinguishable from chromatograms generated for noninfected plants (Fig. 1), showing that the increase in SAG concentration was specific for $V$. longisporum.

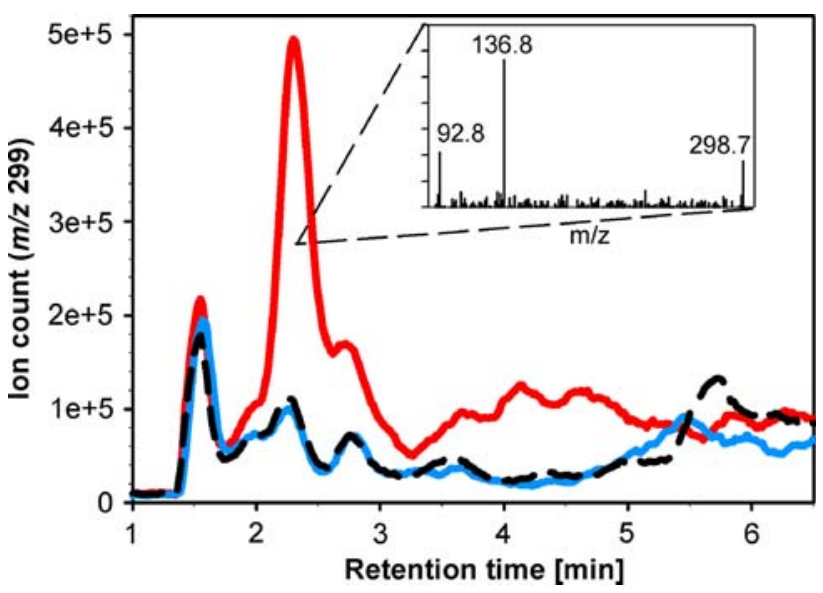

Fig. 1 Selected ion chromatogram of salicylic acid glucoside (SAG) in xylem sap. Xylem sap of healthy plants (black dashed line), Verticillium longisporum-infected plants (red line) and of Verticillium dahliae-infected plants (blue line) was analysed by HPLC-MS in selected ion monitoring mode. Ion chromatograms of SAG $(\mathrm{m} / z, 299$, $[\mathrm{M}-\mathrm{H}]^{-}$) and product ion spectra of $\mathrm{m} / z, 299$ are shown (Colour figure online) 
Relationship between SA and SAG concentrations, disease symptoms and fungal biomass

Because SAG is formed from SA by glucosyl transferase (Yalpani et al. 1992), in addition to SAG we also determined the concentration of SA in xylem sap and shoot extracts from individual plants. Plants infected with $V$. longisporum and healthy controls were harvested at 28 dpi, xylem sap was obtained from the root and hypocotyl part of each plant, and the shoots were subjected to acidic methanol/chloroform/water extraction. Xylem sap samples and shoot extracts were analysed by HPLC-MS, using multiple reaction monitoring on a triple-quadrupole. Large differences in SA and SAG concentrations among individual infected plants indicated that SA and SAG were affected by the level of infection, which is notoriously variable (Eynck et al. 2007). Infected plants contained 0.2-1.5 $\mu \mathrm{M}$ SAG in xylem sap and 2-189 nmol/g dry weight in shoots. Healthy plants contained only $0.06-0.11 \mu \mathrm{M}$ SAG in xylem sap and $0.51-1.48 \mathrm{nmol} / \mathrm{g}$ dry weight in shoots. The concentrations of SA and its glucoside were correlated strongly both in shoot tissue and xylem sap, the levels of SA being about twice as high as the levels of SAG (Fig. 2a, b). Both SA and SAG levels were also correlated between xylem sap and shoot extracts (data for SAG are shown in Fig. 2c).

As stunting is the most prominent symptom of the infection of rapid-cycling rape with $V$. longisporum in the greenhouse, we investigated the relationships between SA and SAG levels and shoot length. SAG and SA concentrations in both shoot extracts and xylem sap were correlated with a reduction in shoot length (Fig. 3).

Because disease symptoms are caused, directly or indirectly, by fungal colonisation, we determined the amount of fungal DNA in the hypocotyls of individual infected plants at $28 \mathrm{dpi}$ and compared these values to the SAG concentration in the shoot and to shoot length. A strong correlation between SAG levels in the shoot and the amount of $V$. longisporum DNA in hypocotyls was found (Fig. 4a). The reduction in shoot length and the amount of $V$. longisporum DNA were also correlated, although the correlation was less pronounced (Fig. 4b).

Changes in concentrations of SA, SAG, JA, and ABA in xylem sap during infection of $B$. napus with $V$. longisporum

The work of Johansson et al. (2006) hinted at the involvement of JA, and possibly ABA, pathways in the infection of $A$. thaliana with $V$. longisporum. We therefore simultaneously determined the concentrations of SA, SAG, $\mathrm{JA}$ and ABA in xylem sap collected from B. napus plants at 14, 21, 28, and $35 \mathrm{dpi}$. Because plants were too small to deliver sufficient amounts of xylem sap at early time
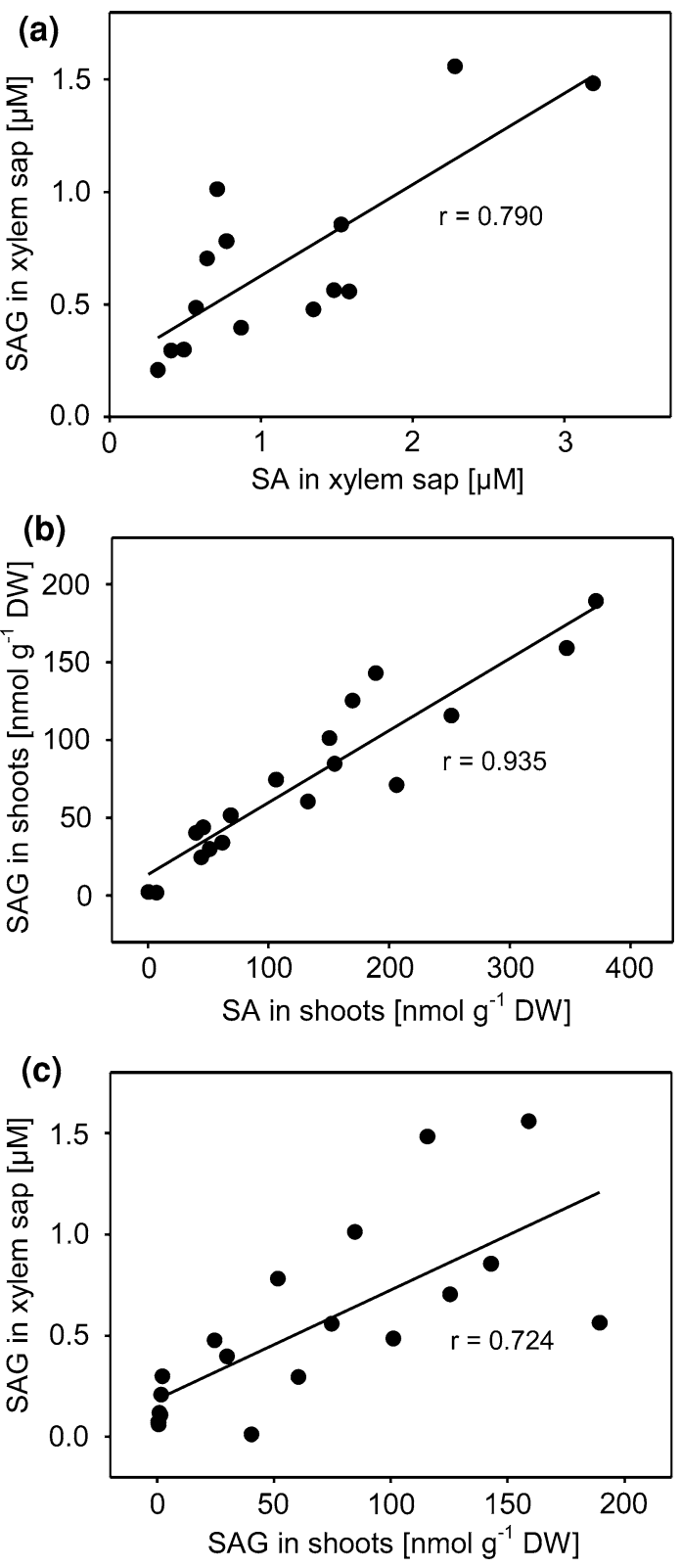

Fig. 2 Comparison of the concentrations of salicylic acid (SA) and SAG in xylem sap and shoot extracts. Xylem sap from V. longisporuminfected single plants was analysed 28 days post infection (dpi). Pearson correlation is given $(r)$, all correlations are significant at $P \leq 0.01$ (1-tailed $t$ test). a SA versus SAG in xylem sap, b SA versus SAG in shoots, $\mathbf{c}$ SAG in shoots versus SAG in xylem sap

points, samples from four plants were pooled for each analysis. Salicylic acid glucoside, SA, JA, and ABA concentrations were determined by injecting filtered xylem sap directly onto the HPLC column. The results are shown in Fig. 5. For both healthy plants and $V$. dahliae-infected plants, SAG concentrations remained at the same level (20-40 nM) over the course of the experiment. After infection with $V$. longisporum, SAG concentrations 
Fig. 3 Concentrations of SAG $(\mathbf{a}, \mathbf{c})$ and SA (b, d) in shoot extracts (a, b) and xylem sap $(\mathbf{c}, \mathbf{d})$ in relation to shoot length. Xylem sap from

V. longisporum-infected single plants was analysed 28 dpi. Pearson correlation is given $(r)$, all correlations are significant at $P \leq 0.05$ (1-tailed $t$ test)
Fig. 4 Concentrations of SAG in shoot extracts (a) and shoot length (b) in relation to the amount of fungal DNA in the hypocotyl. Shoot extracts from individual $V$. longisporuminfected plants were analysed 28 dpi; fungal DNA (V. l.-DNA) in hypocotyls was determined by real-time PCR. Pearson correlation is given $(r)$, all correlations are significant at $P \leq 0.01$ (1-tailed $t$ test)
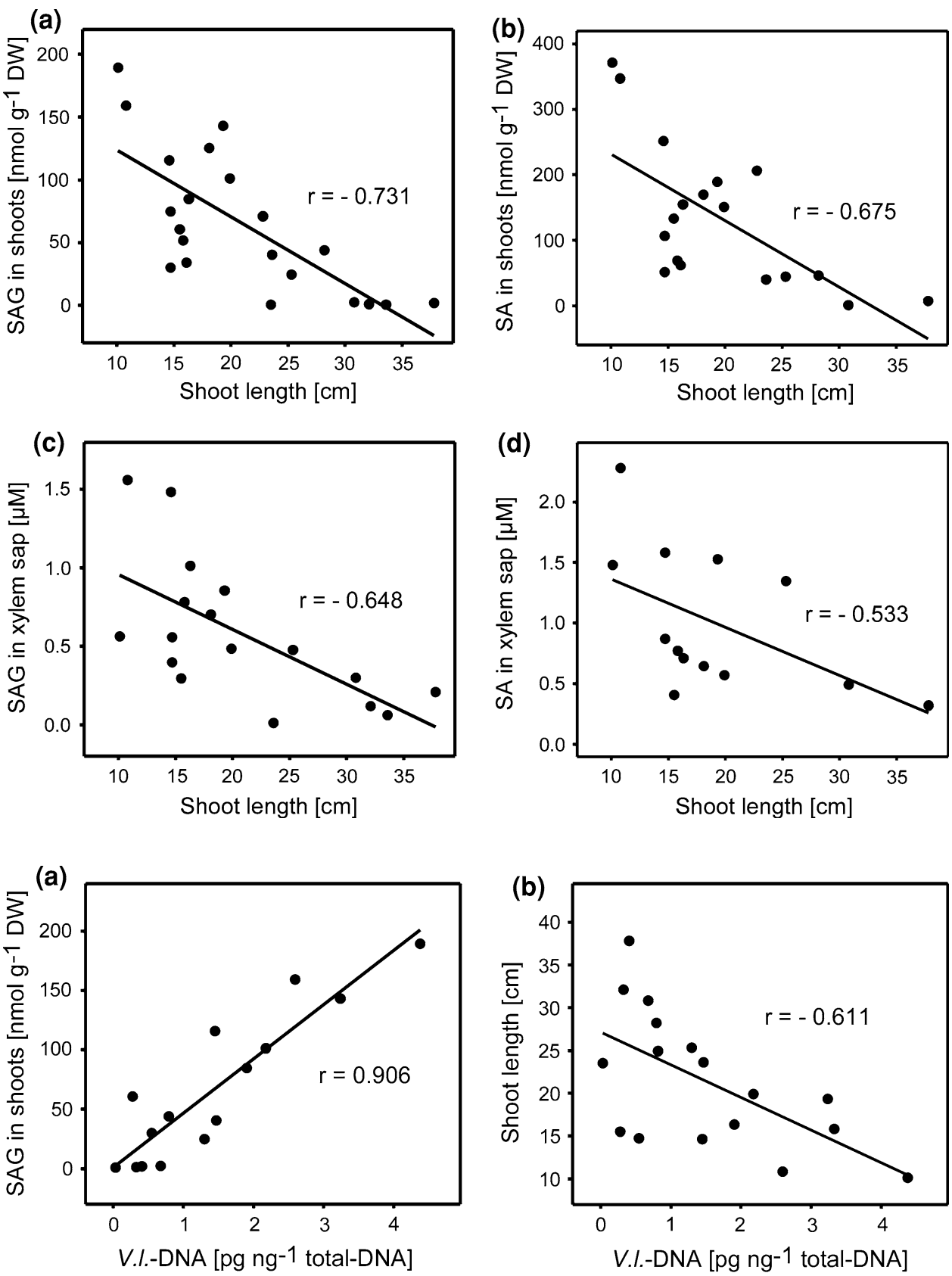

increased strongly at 14 dpi or earlier and declined gradually thereafter, while remaining significantly higher than in the controls throughout the whole experiment. Similarly, SA concentration in xylem sap was significantly higher as compared to uninfected plants at all time points in plants infected with $V$. longisporum but not with $V$. dahliae (Fig. 5a). The ratio of SA concentration in xylem sap of $V$. longisporum-infected versus healthy plants increased from 4 to 9 between 14 and $35 \mathrm{dpi}$, because the gradual decrease in SA concentration was less pronounced in infected plants than in healthy plants (Fig. 5b).

The levels of JA and ABA in xylem sap increased with plant age, but did not differ significantly between
V. longisporum-infected, $V$. dahliae-infected and healthy plants (Fig. 5c, d).

\section{Discussion}

We detected SA and SAG in xylem sap of B. napus and showed that their concentrations increased after infection with $V$. longisporum. High concentrations of SA occur in phloem sap after pathogen attack (Métraux et al. 1990; Rasmussen et al. 1991) but neither free endogenous SA nor its derivatives have been reported from xylem sap. A recent study of the re-distribution of $\left[{ }^{14} \mathrm{C}\right]$-labelled SA (Rocher 
Fig. 5 Concentrations of SAG, SA, jasmonic acid (JA) and abscisic acid (ABA) in xylem sap of $V$. longisporum- and V. dahliae-infected Brassica napus plants. Analysis of xylem sap from healthy plants (white), $V$. longisporum-infected plants (black) and V. dahliae-infected plants (hatched) at different time points after infection is shown. Each data point represents an average of five samples (each sample pooled from four plants). Error bars Standard deviation.

* Significant differences $(P \leq 0.05)$ between xylem sap samples from $V$. longisporuminfected plants and both remaining samples (mock- and V. dahliae-infected). a SAG, b SA, c JA, d ABA

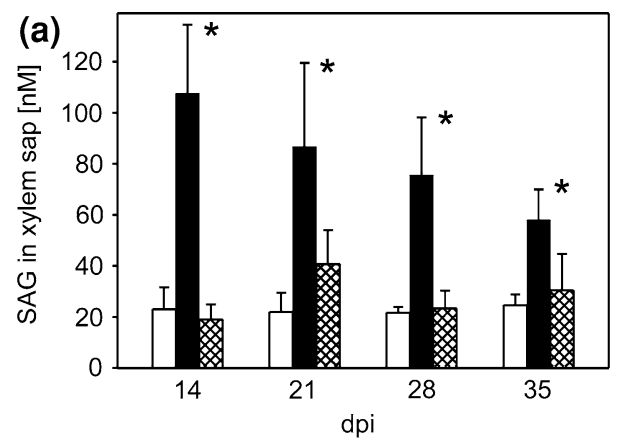

(b)

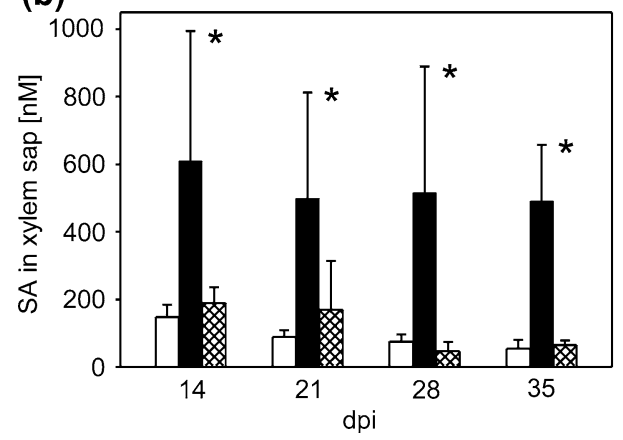

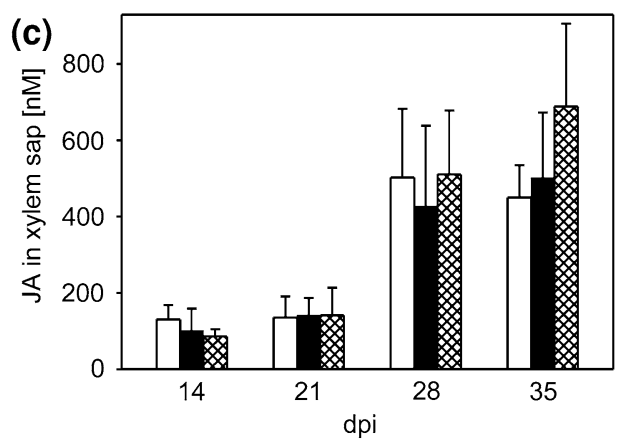

(d)

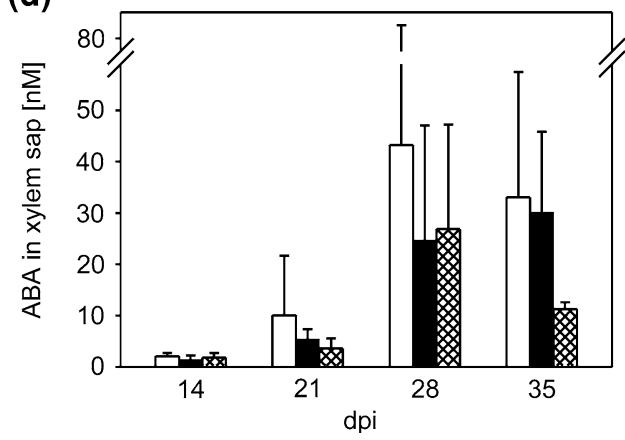

et al. 2006) inspired a hypothesis that the transpiration stream contributes to long-distance transport of SA in plants, but an attempt to detect SA in xylem sap by these latter authors failed.

In principle, the SA and SAG found in xylem sap could have originated from contamination with other tissues. Pollution with cytoplasm from damaged cells appeared negligible because activity of malate dehydrogenase was undetectable, but contamination with phloem sap cannot be excluded. Sucrose is a biomarker for phloem; while present in only trace quantities in xylem sap (Nakamura et al. 2008), sucrose concentrations of 0.98 and $1.34 \mathrm{M}$ were reported for phloem sap of B. napus (Lohaus and Moellers 2000). Comparison of these values with our results $(2.17 \pm 1.04 \mathrm{mM}$ of sucrose plus glucose for infected plants, $3.10 \pm 0.31 \mathrm{mM}$ for uninfected controls) indicates that xylem sap contained less then $0.3 \%$ phloem sap. The concentration of free glucose in xylem sap was $1.31 \pm 0.53 \mathrm{mM}$ (controls) and $1.30 \pm 0.08 \mathrm{mM}$ (infected plants), while Nakamura et al. (2008) reported $0.30 \mathrm{mM}$. Since we assumed that all glucose in xylem originated from hydrolysed sucrose, the contamination of xylem sap with phloem sap was probably even lower than the estimate given above.

Salicylic acid glucoside is formed by glucosyl transferase in a concentration-dependent manner (Dean et al. 2005). Because glycosylation is an intracellular process, SAG must be transported into xylem from the surrounding tissues. Similar ratios of SAG and SA in shoot tissue and in xylem sap (Fig. 2a, b) indicate that both metabolites are secreted into xylem with the same efficiency, contrasting with the selective transport of SAG into the vacuole (Dean and Mills 2004). Recent findings that the suppression of SA glycosylation in rice by gene silencing reduced the development of resistance (Umemura et al. 2008) indicates that SAG may play a central role in SAR. Overexpression of SA glucosyl transferase in Arabidopsis, however, increased susceptibility to a bacterial pathogen (Song et al. 2008). This contradiction was resolved by finding that SA and SAG in transgenic plants decreased rather than increased due to increased activity of SA methyl transferase (Song et al. 2008).

Many biologically active secondary metabolites synthesised in the roots are transported acropetally with the transpiration stream (Satoh 2006). Abscisic acid is synthesised during drought stress in roots and transported to the shoots, where it triggers a drought response (Hartung et al. 2002). Zeatins are also carried from roots to shoots (Kato et al. 2002; Kuroha et al. 2005). According to our data, in B. napus, SA and SAG are transported in the same manner. Our results proved Rocher et al. (2006) right in their prediction that "endogenous SA levels in xylem sap should be far from negligible in infected plants". In this context, it is interesting to note that the specific activity of SA glucosyl transferase in oat was ten-times higher in roots than in shoots (Yalpani et al. 1992).

In A. thaliana, the role of the SA pathway in response to Verticillium infection has been studied by Veronese et al. (2003), Tjamos et al. (2005) and Johansson et al. (2006). The first two groups worked with $V$. dahliae and obtained 
contradictory results regarding the expression of SAdependent pathogenesis-related genes. Because the taxonomical status of $V$. longisporum is controversial, and its separation from $V$. dahliae is sometimes difficult, one of the strains used in these studies might actually have been V. longisporum (A. von Tiedemann, unpublished results). The third laboratory worked with $V$. longisporum and described the induction of $P R-1$ and $P R-2$ as early as $7 \mathrm{dpi}$, but the SA pathway was found not to be involved in the defence because plants with defective SA pathway did not exhibit enhanced susceptibility. In our experiments, the amount of SAG in the shoot was correlated with fungal biomass in the hypocotyl (Fig. 4a). Similar correlations were found for SA in shoot extracts and fungal DNA, and for SA and SAG in xylem sap and DNA (data not shown). Furthermore, both SA and SAG concentrations were correlated negatively with shoot length (Fig. 3). The determination of SA and SAG concentrations in A. thaliana after infection might help assess the suitability of the A. thaliana/V. longisporum pathosystem as a model for Verticillium disease of oilseed rape.

$V$. dahliae-infected plants were indistinguishable from uninfected controls both in SA and SAG levels and in their phenotype (Fig. 5a, b). The lack of disease symptoms in $B$. napus infected with $V$. dahliae has been demonstrated before by Eynck et al. (2007).

Johansson et al. (2006) demonstrated that the JA pathway was involved in the response of A. thaliana to $V$. longisporum, and that $\mathrm{ABA}$ was not required (although an $A B A 2$ mutant was impaired in defence reaction). In our work with B. napus, JA levels in xylem sap increased, and those of SAG decreased with plant age (Fig. 5). This is indicative of an antagonistic relationship between the two pathways (Takahashi et al. 2004). Because infected and healthy plants exhibited similar changes in JA concentration over time, however, aging-related processes were more likely the cause. JA is known to be involved in senescence (Fukushige et al. 2002). Obviously the roles of JA in the defence of Arabidopsis and Brassica against V. longisporum are different, possibly reflecting the fact that Brassica, unlike Arabidopsis, is a natural host for the fungus.

Veronese et al. (2003) showed that ABA (but not SA or JA) acted as a regulator of the A. thaliana response to $V$. dahliae infection (the strain used might actually have been $V$. longisporum). Antagonism between the ABA and SA pathways was demonstrated in Arabidopsis (Yasuda et al. 2008), but in our system the increase in SA concentration caused by $V$. longisporum infection did not affect endogenous ABA levels (Fig. 5).

This work demonstrates for the first time the presence of SA and SAG in xylem sap. These two metabolites thus expand the list of phytohormones transported with the transpiration stream from roots to shoots (Satoh 2006). The biological role of the enhanced levels of SA and SAG in xylem sap after infection with a vascular pathogen remains to be elucidated.

Acknowledgments This research work was part of an interfacultative research unit FOR546 funded by the Deutsche Forschungsgemeinschaft (DFG), Bonn, Germany. We gratefully thank Prof. H. Kneifel (Forschungszentrum Jülich, Jülich, Germany) for providing us with the SAG standard. The technical assistance of P. Bartoschek and M. Bossmann is highly appreciated.

\section{References}

Brandfass C, Karlovsky P (2006) Simultaneous detection of Fusarium culmorum and $F$. graminearum in plant material by duplex PCR with melting curve analysis. BMC Microbiol 6:4

Dean JV, Mills JD (2004) Uptake of salicylic acid 2-O-b-D-glucose into soybean tonoplast vesicles by an ATP-binding cassette transporter-type mechanism. Physiol Plant 120:603-612

Dean JV, Mohammed LA, Fitzpatrick T (2005) The formation, vacuolar localization, and tonoplast transport of salicylic acid glucose conjugates in tobacco cell suspension cultures. Planta 221:287-296

Enyedi AJ, Yalpani N, Silverman P, Raskin I (1992) Localization, conjugation, and function of salicylic acid in tobacco during the hypersensitive reaction to tobacco mosaic virus. Proc Natl Acad Sci USA 89:2480-2484

Eynck C, Koopmann B, Grunewaldt-Stoecker G, Karlovsky P, von Tiedemann A (2007) Differential interactions of Verticillium longisporum and $V$. dahliae with Brassica napus detected with molecular and histological techniques. Eur J Plant Pathol 118:259-274

Fukushige H, Hildebrand D, Gan S (2002) Evidence supporting a role of jasmonic acid in Arabidopsis leaf senescence. Plant Physiol 128:876-884

Hartung W, Sauter A, Hose E (2002) Abscisic acid in the xylem: where does it come from, where does it go to? J Exp Bot 53:2732

Johansson A, Staal J, Dixelius C (2006) Early responses in the Arabidopsis-Verticillium longisporum pathosystem are dependent on NDR1, JA- and ET-associated signals via cytosolic NPR1 and RFO1. Mol Plant Microbe Interact 19:958-969

Kato C, Kato H, Asami T, Yoshida S, Noda H, Kamada H, Satoh S (2002) Involvement of xylem sap zeatin- $O$-glucoside in cucumber shoot greening. Plant Physiol Biochem 40:949-954

Kawano T, Tanaka S, Kadono T, Muto S (2004) Salicylic acid glucoside acts as a slow inducer of oxidative burst in tobacco suspension culture. Z Naturforsch [C] 59:684-692

Kiefer IW, Slusarenko AJ (2003) The pattern of systemic acquired resistance induction within the Arabidopsis rosette in relation to the pattern of translocation. Plant Physiol 132:840-847

Kuroha T, Sakurai M, Satoh S (2005) Squash xylem sap has activities that inhibit proliferation and promote the elongation of tobacco BY-2 cell protoplasts. Plant Physiol Biochem 43:465-471

Loake G, Grant M (2007) Salicylic acid in plant defence-the players and protagonists. Curr Opin Plant Biol 10:466-472

Lohaus G, Moellers C (2000) Phloem transport of amino acids in two Brassica napus $\mathrm{L}$. genotypes and one B. carinata genotype in relation to their seed protein content. Planta 211:833-840

Métraux JP, Signer H, Ryals J, Ward E, Wyss-Benz M, Gaudin J, Raschdorf K, Schmid E, Blum W, Inverardi B (1990) Increase in salicylic acid at the onset of systemic acquired resistance in cucumber. Science 250:1004-1006 
Mölders W, Buchala A, Metraux JP (1996) Transport of salicylic acid in tobacco necrosis virus-infected cucumber plants. Plant Physiol 112:787-792

Nakamura S, Akiyama C, Sasaki T, Hattori H, Chino M (2008) Effect of cadmium on the chemical composition of xylem exudate from oilseed rape plants (Brassica napus L.). Soil Sci Plant Nutr $54: 118-127$

Rasmussen JB, Hammerschmidt R, Zook MN (1991) Systemic induction of salicylic acid accumulation in cucumber after inoculation with Pseudomonas syringae pv syringae. Plant Physiol 97:1342-1347

Rocher F, Chollet JF, Jousse C, Bonnemain JL (2006) Salicylic acid, an ambimobile molecule exhibiting a high ability to accumulate in the phloem. Plant Physiol 141:1684-1693

Satoh S (2006) Organic substances in xylem sap delivered to aboveground organs by the roots. J Plant Res 119:179-187

Scholander PF, Hammel HT, Bradstre ED, Hemmings EA (1965) Sap pressure in vascular plants-negative hydrostatic pressure can be measured in plants. Science 148:339-346

Smith CA, Want EJ, O`Maille G, Abagyan R, Suizdak G (2006) XCMS: processing mass spectrometry data for metabolite profiling using nonlinear peak alignment, matching, and identification. Anal Chem 78:779-787

Song JT, Koo YJ, Seo HS, Kim MC, Choi YD, Kim JH (2008) Overexpression of AtSGT1, an Arabidopsis salicylic acid glucosyltransferase, leads to increased susceptibility to Pseudomonas syringae. Phytochemistry 69:1128-1134

Takahashi H, Kanayama Y, Zheng MS, Kusano T, Hase S, Ikegami M, Shah J (2004) Antagonistic interactions between the SA and JA signaling pathways in Arabidopsis modulate expression of defence genes and gene-for-gene resistance to cucumber mosaic virus. Plant Cell Physiol 45:803-809

Tjamos SE, Flemetakis E, Paplomatas EJ, Katinakis P (2005) Induction of resistance to Verticillium dahliae in Arabidopsis thaliana by the biocontrol agent K-165 and pathogenesis-related proteins gene expression. Mol Plant Microbe Interact 18:555561
Umemura K, Satou J, Iwata M, Uozumi N, Koga J, Kawano T, Koshiba T, Anzai H, Mitomi M (2008) Contribution of salicylic acid glucosyltransferase, OsSGT1, to chemically-induced disease resistance in rice plants. Plant J 57:463-472

Verberne MC, Verpoorte R, Bol JF, Mercado-Blanco J, Linthorst HJ (2000) Overproduction of salicylic acid in plants by bacterial transgenes enhances pathogen resistance. Nat Biotechnol 18:779-783

Vernooij B, Friedrich L, Morse A, Reist R, Kolditz-Jawhar R, Ward E, Uknes S, Kessmann H, Ryals J (1994) Salicylic acid is not the translocated signal responsible for inducing systemic acquired resistance but is required in signal transduction. Plant Cell 6:959-965

Veronese P, Narasimhan ML, Stevenson RA, Zhu JK, Weller SC, Subbarao KV, Bressan RA (2003) Identification of a locus controlling Verticillium disease symptom response in Arabidopsis thaliana. Plant J 35:574-587

Yalpani N, Silverman P, Wilson TM, Kleier DA, Raskin I (1991) Salicylic acid is a systemic signal and an inducer of pathogenesis-related proteins in virus-infected tobacco. Plant Cell 3:809818

Yalpani N, Balke NE, Schulz M (1992) Induction of UDP-glucose: salicylic acid glucosyltransferase in oat roots. Plant Physiol 100:1114-1119

Yasuda M, Ishikawa A, Jikumaru Y, Seki M, Umezawa T, Asami T, Maruyama-Nakashita A, Kudo T, Shinozaki K, Yoshida S, Nakashita H (2008) Antagonistic interaction between systemic acquired resistance and the abscisic acid-mediated abiotic stress response in Arabidopsis. Plant Cell 20:1678-1692

Zeise K, von Tiedemann A (2001) Morphological and physiological differentiation among vegetative compatibility groups of Verticillium dahliae in relation to Verticillium longisporum. J Phytopathol 149:469-475

Zhou L, Hu Q, Johansson A, Dixelius C (2006) Verticillium longisporum and Verticillium dahliae: infection and disease in Brassica napus. Plant Pathol 55:137-144 